\title{
O uso do canabidiol como medicamento no Brasil para tratar doenças crônicas
}

\author{
The use of cannabidiol as medicine in Brazil to treat chronic diseases
}

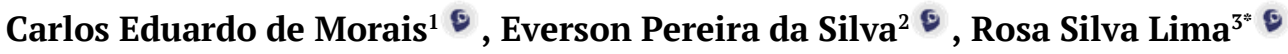 \\ 1,35aculdade do Instituto Brasil (FIBRA), Instituto Brasil de Ciência \& Tecnologia (IBCT), Anápolis, Goiás, Brasil ${ }^{2}$ Universidade Paulista \\ (Unip-EAD), Anápolis, Goiás, Brasil. *Autor para correspondência. E-mail: rosinha.576@hotmail.com
}

\begin{abstract}
Resumo: Introdução: No Brasil a maconha teve início junto com sua descoberta, no país o tema sempre trouxe muita discussão, e uma planta exótica, nativa da Ásia, utilizada durante muitos séculos para diversos fins, como rituais religiosos e práticas medicinais. Os estudos das substancias isoladas da cannabis ativa começaram por volta de 1960, sendo descoberta varias substancias dentre elas o (THC) e (CDB) destacando o canabidiol (CBD) substância não alucinógena. E ainda apresenta inúmeras propriedades farmacológicas. Segundo a legislação a maconha e considerada uma droga ilícita proibida no pais, não sendo permitido a sua posse, aquisição e transporte. Antes da vigência da RDC No 327 de 9 de dezembro de 2019 onde ficou autorizado no Brasil a comercialização de produtos contendo (THC) e (CBD), os pacientes que precisavam utilizar os ativos da planta para tratamento eram obrigados a utilizar meios jurídicos para conseguir autorização de importação dos medicamentos com substancias da maconha. Portanto o objetivo desta pesquisa foi realizar uma revisão bibliográfica em artigos digitais e livros virtuais encontrados no Google acadêmico, em plataformas de bases de dados Scientific Electronic Library Online (SCIELO), Biblioteca Virtual em Saúde (BVS). Através desta, o trabalho procurou descrever o conceito geral da maconha, buscando definir o seu conceito ao longo do tema proposto, bem como elencar os benefícios ao organismo humano e possíveis ações terapêuticas para as doenças, não deixando de destacar os malefícios decorrentes do seu uso. Revisão: São apresentadas informações de uma pesquisa de caráter exploratório descritivo onde são compiladas informações sobre o conhecimento de alunos de 2 escolas públicas da cidade de Anápolis-GO, através de um questionário onde os alunos responderam perguntas referentes aos compostos CBD e THC. Discussão: Foi possível entender através da elaboração desta revisão que a Cannabis sativa (maconha) já vem sendo usada a milhares de anos como método terapêutico, e que somente após ter sido catalogadas as substancias da planta, foi possível a sua utilização como método adicional a terapêutica tradicional. Considerações finais: Apesar da maconha ter trazido vários problemas para as autoridades devido o seu uso recreativo, o uso das substancias isoladas trás vários benefícios aos pacientes doentes graves, utilizado como método adicional ao tratamento convencional.
\end{abstract}

Palavras-chave: Cannabis sativa, canabidiol, medicamento, efeito terapêutico, tratamento.

\begin{abstract}
Introduction: In Brazil marijuana began along with its discovery, in the country the theme has always brought much discussion, and an exotic plant, native to Asia, used for many centuries for various purposes, such as religious rituals and medicinal practices. Studies of the substances isolated from active cannabis began around 1960, and several substances were discovered, among them the (THC) and (CDB) highlighting cannabidiol (CBD) non-hallucinogenic substance. It also has numerous pharmacological properties. According to the legislation marijuana and considered an illicit drug prohibited in the country, not being allowed possession, acquisition and transport. Before the validity of DRC $\mathrm{N}^{\circ} 327$ of December 9, 2019 where was authorized in Brazil the commercialization of products containing (THC) and (CBD), patients who needed to use the plant's assets for treatment were required to use legal means to obtain authorization to import the drugs with marijuana substances. Therefore, the objective of this research was to carry out a bibliographic review in digital articles and virtual books found in academic Google, in databases platforms Scientific Electronic Library Online (SCIELO), Biblioteca Virtual em Saúde (BVS). Through this, the work sought to describe the general concept of marijuana, seeking to define its concept throughout the proposed theme, as well as to list the benefits to the human body and possible therapeutic actions for diseases, while highlighting the harm caused by its use. Review: Information is presented from a descriptive exploratory research where information on the knowledge of students from 2 public schools in the city of Anápolis is compiled-GO, through a questionnaire where the students answered questions related to the compounds CBD and THC. Discussion: It was possible to understand through the elaboration of this review that Cannabis sativa (marijuana) has been used for thousands of years as a
\end{abstract}


therapeutic method, and that only after having catalogued the substances of the plant, it has been possible to use it as an additional method to traditional therapy. Final considerations: Although marijuana has brought several problems to authorities due to its recreational use, the use of isolated substances brings several benefits to severely ill patients, used as an additional method to conventional treatment.

Keywords: Cannabis sativa, cannabidiol, medicine, therapeutic effect, treatment.

\section{Introdução}

A Cannabis sp. Popularizada como maconha, é um arbusto pertencente à família das Cannabaceae, compondo-se com aproximadamente quatrocentas substâncias químicas, dentre esses compostos sessenta são os chamados canabinoides, conforme pesquisa do Instituto Nacional de Saúde. Nativa do continente Asiático era utilizada para confeccionar cordas e tecidos, mas também descobriram através de manuscritos chineses o seu uso para fins terapêuticos e praticas religiosa. Durante milênios foi utilizada por metade do mundo, sua chegada à América do Norte se deu através de Cristóvão Colombo para fins comerciais. No entanto somente, no início do século XX se tornou comum o seu uso recreativo, foi considerado ilegal por ser tão prejudicial quanto a cocaína e a heroína (Carlini et al., 2005).

Os primeiros indícios do seu uso foram registrados a aproximadamente dois mil e quinhentos A. C. no continente Asiático. Local que provavelmente era cultivada e utilizada como método terapêutico por curandeiros no passado (Pamplona, 2014).

A Cannabis sp. é um vegetal da espécie botânica Cannabaceae. Essa denominação faz referência à três diferentes plantas: Cannabis sativa, $C$. indica e $C$. ruderalis, que se distinguem por seus hábitos de desenvolvimento, por aspectos morfológicos e pela concentração de princípios ativos (Gonçalves \& Schlichting, 2014).

A entrada da planta no Brasil ocorreu em meados do ano de 1500 trazida pelos escravos africanos, na época do descobrimento, era usada apenas com finalidade recreativa, visto que nesta época não se conhecia o seu potencial terapêutico. O vegetal era conhecido pelos escravos como cânhamo da Índia. A origem da palavra "maconha" ocorreu por meio de anagrama "cânhamo" que consiste na alteração da ordem das letras para formar outra (Carlini, 2006).

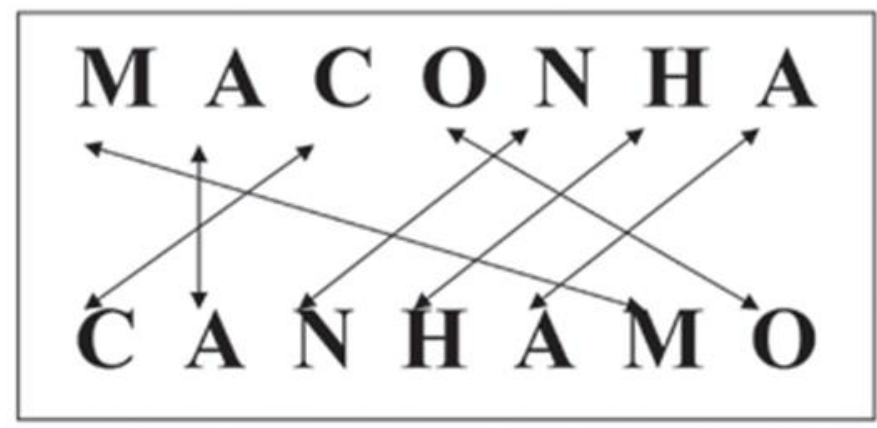

Figura 1. Maconha anagrama da palavra cânhamo. Fonte: Carlini (2006).

Os principais substratos da planta foram descobertos e catalogados na década de 60 por profissionais multidisciplinares, onde em meio a muitos, se destacaram o delta nove-tetrahidrocanabidiol que apesar de seus efeitos terapêuticos e o único a possuir efeito psicoativo alucinógeno, enquanto que o canabidiol não possui tais efeitos, entretanto exerce várias atividades terapêuticas (Gontiès \& Araújo, 2003).

A ação terapêutica da Cannabis sativa foi claramente elucidada após muitos estudos. A maconha e composta por cerca de 400 compostos químicos, com cerca de 60 canabinoides, que são princípios ativos distintos (Monteiro, 2014).

Por apresentarem propriedades medicinais dois se destacam: o tetrahidrocanabinol (THC) e o canabidiol (CDB). Segundo estudos, esse importante componente da droga (THC), os ligantes endógenos e os canabinoides, em pacientes oncológicos possuem atividade analgésica, antitumoral, estimulante do apetite, propiciam um relaxamento muscular e diminuição da insônia (Monteiro, 2014).

Mesmo com sua atividade terapêutica comprovada tornou-se um desafio aprovar o uso para fins medicinais deles, em especial, do CBD. As autoridades temem que a liberação do uso terapêutico dos canabinoides poderá favorecer o uso da droga de forma recreativa pelos usuários (Carlini et al., 2005). 
A Agência reguladora (ANVISA) autorizou no Brasil o uso medicinal do CBD por importação para casos específicos, entretanto, deverá ser exigido receita, laudos médicos e termo de responsabilidade (Brucki et al., 2015).

Devido ao CBD ainda não ser registrado na ANVISA, seu uso pode ser liberado através do uso compassivo, definido pela prescrição de uma substância destinada a pacientes doentes graves e ausentes de alternativa terapêutica com produtos já registrados (Matos et al., 2017).

Após a vigência da RDC No 327 de 9 de dezembro de 2019 fica liberado a fabricação a partir de importação de matérias primas extraídas da Cannabis sativa, de produtos para fins medicinais utilizados apenas por via oral ou nasal. Podendo ser comercializado por farmácias e drogarias devidamente autorizadas. O paciente terá acesso ao medicamento mediante apresentação da prescrição médica. Os produtos contendo ativos da Cannabis deve possuir em maior quantidade o canabidiol (CBD) e uma quantidade inferior a $0,2 \%$ de tetrahidrocanabinol (THC), os que poderão ter teor acima de $0,2 \%$ devem ser destinados exclusivamente a pacientes sem alternativas terapêuticas ou com situações clinicas irreversíveis ou terminais (Ministério da Saúde, 2019).

Embora os compostos do vegetal produzam efeitos sobre um determinado número de órgãos, dentre os quais estão incluídos o sistema imunológico e reprodutivo, os efeitos terapêuticos apurados estão relacionados ao sistema nervoso central. Esses efeitos, em seres humanos correspondem à: analgesia (Brooks, 2002), mudança de humor, atua aumentando o apetite em pessoas tratados com quimioterapia (pacientes portadores de HIV e câncer) (Mechoulam, 1973), atividades psicomotoras com alteração, na percepção, na cognição, na memória, no controle da espasticidade em pacientes esclerótico (Baker et al., 2000).

o $\Delta$ 9-Tetrahidrocanabidiol (Figura 2) é o canabinoide que provoca efeitos psicóticos em pessoas vulneráveis, responsável pelo aumento do fluxo pré-sináptico de dopamina no córtex pré-frontal medial (Crippa et al., 2005).

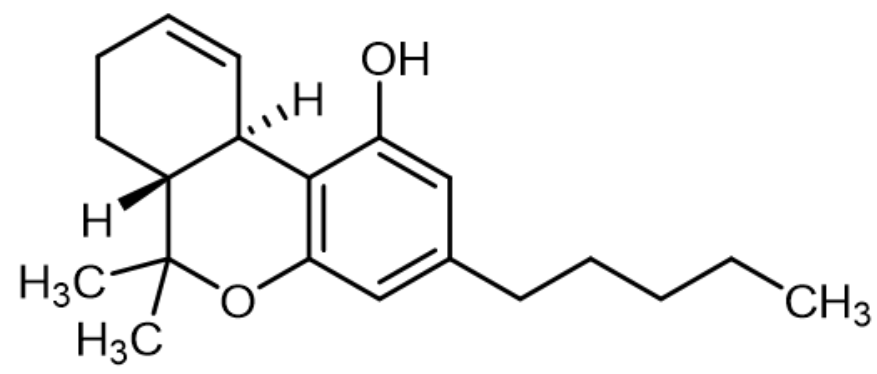

Figura 2. (6aR,10aR)-6,6-dimethyl-3-pentyl-6a,7,8,10a-tetrahydro-6H-benzo[c]chromen-1-ol (THC). Fonte: Chemdraw professional 16.0

Encontrado em até $40 \%$ dos extratos da planta, o CBD possui grande importância por ser o principal componente não alucinógeno da Cannabis sativa, já o $\Delta$ 9-THC tem a capacidade de produzir efeito psicoativo alucinógeno (Campos, 2012).

Esses dois compostos atuam como antagônicos, uma vez que são altamente competitivos. Enquanto o $\triangle 9$-THC atua gerando sensação de euforia, o CBD atua bloqueando e reduzindo a expressão do humor (Figura 3), (Bergeret \& Leblanc, 1991).

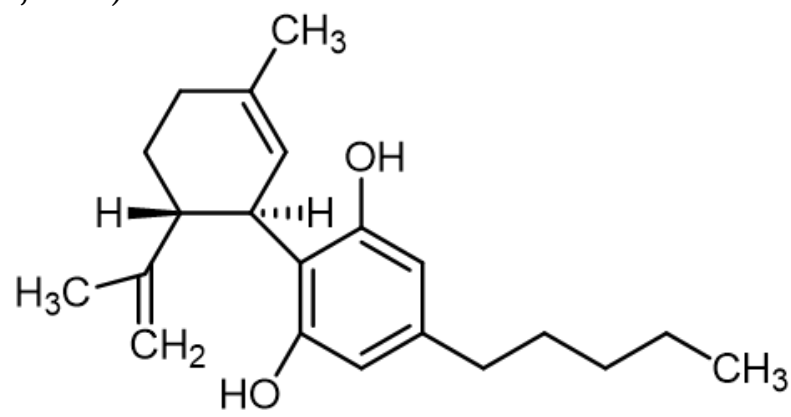

Figura 3. (1'R,2'R)-5'-metil-4-pentil-2'-(prop-1-en-2-il)-1',2',3',4'-tetrahidro-[1,1'-bifenil]-2,6-diol (CBD). Fonte: Chemdraw professional 16.0 .

Os dois são destacados por ter propriedades medicinais elucidadas, tanto o tetrahidrocanabidiol (THC) quanto o Canabidiol (CBD). Conforme constatado, os ligantes endógenos e os canabinoides, por atribuição de sua formulação, acabam por proporcionar efeito analgésico, antitumoral, aumentando o apetite, reduzindo a insônia e proporcionando relaxamento muscular (Miranda, 2016). 


\section{Revisão}

Este trabalho foi realizado mediante pesquisa de revisão bibliográfica, para a composição deste estudo foram utilizados artigos, dissertações, teses e livros para obtenção de informações sobre o uso dos princípios ativos da maconha (THC e CBD) como opção de tratamento para doenças crônicas e degenerativas.

Realizou-se consultas em bases de dados bibliográficas: Base de Científica Electronic Library Online (SCIELO), Science Direct, Pumed.

Em relação a opção de escolha dos artigos, foram selecionados os publicados entre 2000 a 2020 em periódicos nacionais e internacionais, nos quais foram avaliadas as seguintes palavras chaves: maconha, canabidiol, $\Delta$ 9-Tetrahidrocanabidiol, liberação e uso controlado.

Para realizar a contextualização histórica, utilizou-se referencias antes do ano de 2000, pois o uso da maconha é datado deste antes de cristo em periódicos chineses.

Os artigos descartados foram os que não se relacionaram com o tema proposto pelo trabalho onde foi aplicado leitura explorativa de todo material selecionado (leitura rápida, objetiva para avaliar a relevância do trabalho em relação ao tema). Leitura seletiva (segunda leitura do material). Coleta de informações a partir da leitura dos artigos científicos, dissertações e das teses, com o intuito de obter informação sobre o tema explorado.

Realizou-se também uma pesquisa exploratória de caráter descritivo, que permitiu a compilação de informações, através de recursos metodológicos, possuindo uma abordagem qualitativa por meio de um questionário contendo 5 questões sobre os compostos CBD e THC, com 180 alunos acima de 18 anos de 2 escolas públicas da cidade de Anápolis-GO. As questões trabalhadas neste estudo foram: 1) Você conhece o canabidiol e o THC? 2) Qual é a diferença entre a estrutura do THC E CBD?; 3) Para quais doenças esses compostos podem ser usados?; 4) Quais são os problemas que a liberação da venda desses compostos pode acarretar a população jovem?; 5) Canabidiol no brasil: legalizado ou não? Você concorda com essa liberação por quê?

\section{Discussão}

A realização de uma leitura explorativa se fez necessário, com o objetivo de organizar as informações contidas nos artigos selecionados e identificar tópicos propostos nos objetivos desta revisão.

Fez-se possível entender através da elaboração desta revisão que a Cannabis sativa (maconha) já vem sendo usada a milhares de anos como método terapêutico por curandeiros, compreendendo que somente após ter sido catalogados os substratos da planta por meio de estudos destacando o (THC) e o (CBD) princípios ativos da planta destinado ao tratamento de patologias, onde o método tradicional não traz o benefício esperado, podendo ser utilizado como método adicional ao tratamento proposto como associação, trazendo benefícios a pacientes doente grave ou terminal.

Mesmo com sua atividade terapêutica comprovada, no Brasil o uso só e permitido mediante receita, laudos médicos e termos de responsabilidade.

Em países com legislações mais flexíveis atualmente a administração clínica da maconha e utilizada para tratamento de câncer, regulação do aparelho gastrintestinal, redução de náuseas e vômitos. A ação anestésica do THC e alcançada após uso oral causando alivio da dor em pacientes após procedimento quimioterápico.

Diante das diversas atribuições do CBD, se sobressai sua atuação como anticonvulsivante para epiléticos, nas confusões dos movimentos distônico, no tratamento de sintomas do mal de Huntington (uma doença heredodegenerativa do sistema nervoso central), também auxilia nos casos de distúrbios do sono e com grande eficácia como antipsicóticos (Conrad, 2001).

Diversos outros aspectos favoráveis acerca da provável utilização do CBD, e que existem diversos estudos in vivo e in vitro da aplicação do CBD para tratamento sob um extenso espectro de concentrações, sem a detecção de efeitos colaterais e tóxicos relevantes (Conrad et al., 20012).

Apesar disso, a ministração aguda de CBD por diferentes vias não provocou nenhum resultado tóxico importante em humanos. Além disso foi assistido que a administração crônica do CBD por um mês em pessoas voluntárias sadias (doses diárias com concentrações entre 10 a $400 \mathrm{mg}$ ), não promoveu nenhuma alteração significativa em exames psiquiátricos, neurológicos ou clínicos (Cunha et al., 1980). 
Através da análise das estruturas químicas dos compostos foi possível compreender que são antagônicos competitivos enquanto o (THC) atua gerando sensação de euforia o (CBD) traz redução do humor, mas por atribuição da sua formulação proporcionam muitos efeitos benéficos para o paciente.

Dos 180 alunos entrevistados, $80 \%$ que responderam a pesquisa, apresentaram um conhecimento bem expressivo sobre os compostos derivados da Cannabis sativa.

Quando perguntado se eles conheciam o CBD e THC as respostas foram sim, são compostos derivados da maconha. De 180 alunos, apenas 15 não souberam responder a pergunta e deixaram em branco.

Os alunos do $3^{\circ}$ ano do ensino médio, estudam a química orgânica e por isso, tiveram facilidade em responder à pergunta sobre a diferença entre as estruturas do THC e CBD (em anexo), todos os 180 explicaram que no THC tem a função éter e no CBD o grupamento é a hidroxila do fenol, 100 alunos ainda conseguiram explicar que os efeitos biológicos do CBD são melhores e menos alucinógenos. E que devido aos efeitos terapêuticos o CBD foi escolhido, para uso no tratamento de doenças crônicas, epilepsia, autismo, Parkinson, ansiedade, tecidos moles, neoplasias malignas, depressão, paralisia cerebral e esclerose múltipla.

Quanto a questão da liberação da venda do medicamento a base de CBD, 120 alunos responderam, que a liberação com a receita controlada não há nenhum problema, porque o CBD tem efeitos colaterais mais brandos do que o THC, sendo, portanto, essencial para as pessoas que precisam.

O uso da Cannabis sp. é muito comum entre a população jovem, por isso as respostas quanto aos problemas que eventualmente a venda desse composto poderá causar a população jovem, 90 alunos disseram que é benéfico e que não tem problema para a saúde, então não veem nenhum problema. Pois o uso da maconha é ilegal no Brasil e mesmo assim é de fácil acesso pelos jovens e até mesmo adultos. Alegaram também que a maconha é medicinal, sendo assim, os compostos derivados dela deveriam ser vendidos sem restrições.

Percebeu-se com essa pesquisa que muitos jovens ainda são leigos quanto aos efeitos alucinógenos da Cannabis sp. no sistema nervoso e também psicotrópicos. Desconhecem os perigos da dependência química bem como os efeitos colaterais do seu uso, os problemas familiares, o isolamento social, o desinteresse escolar acompanhado de sono excessivo e problemas cognitivos.

\section{Considerações finais}

Apesar de ser uma planta polêmica possui inúmeras substâncias, sendo várias benéficas e com propriedades terapêuticas comprovadas a maconha vem sendo cultivada pelos povos antigos e que já possuíam o hábito de utilizá-la para fins medicinais caseiros, estudos feitos nas mesmas de maneira mais aprofundada onde foram de grande valia e muito importantes para a descoberta dos chamados canabinoides dentre os 60 encontrados se destacaram o (THC) e o (CBD) os quais trouxeram vários benéficos para tratar doentes graves os quais já estavam com a terapia convencional sem efeito desejado, onde o método tradicional não traz o benefício esperado, podendo ser utilizado como método adicional ao tratamento proposto como associação, trazendo benefícios a pacientes doente grave ou terminal.

No Brasil observa-se que desde sempre o uso desta substância não é aceito pelas autoridades devido a grandes problemas que o uso recreativo da planta trouxe para o país, trazendo uma compreensão equivocada do uso dos seus compostos separados e em doses reguladas, por isso os pacientes que necessitam usar como meio profilático deveriam recorrer a meios judiciais para conseguir o tratamento, somente após a publicação da RDC No 327 de 9 de dezembro de 2019, ficou autorizado no Brasil a importação de ativos isolados extraídas da Cannabis sativa para a fabricação de medicamentos contendo os ativos isolados da maconha (THC) e (CBD) para fins terapêuticos.

O conhecimento dos jovens sobre o assunto não é suficiente para entender que a maconha tem efeitos psicotrópicos e não faz bem as pessoas que gozam de boa saúde, em muitas respostas da pesquisa percebeuse que eles acham a maconha terapêutica. É necessário que o governo faça campanhas de esclarecimento sobre a liberação dos produtos derivados da maconha e desenvolva palestras com profissionais qualificados em escolas e instituições esclarecendo de forma mais precisa aos adolescentes e jovens, sobre os perigos e consequências do uso não medicinal dos constituintes da maconha. 


\section{Agradecimentos}

Agradecemos a Faculdade FIBRA pelo apoio dado a produção do artigo, aos alunos da $3^{\circ}$ série do Ensino Médio pela participação na pesquisa e em responder o questionário.

\section{Referências}

Baker, D., Pryce, G., Croxford, J. L., Brown, P., Pertwee, R. G., Huffman, J. W., \& Layward, L. 2000. Cannabinoids control spasticity and tremor in a multiple sclerosis model. Nature, 404, 84.

Ministério da Saúde. 2019. Resolução da diretoria colegiada - RDC $N^{o}$ 327, de 9 de dezembro de 2019. Acesso em: 04 de jun 2020.

Brooks, J. W. 2002. Cannabinoids and analgesia. Current Anesthesia \& Critical Care, 13(4), 215-220.

Brucki, S. M. D., Frota, N. A., Schestatsky, P., Souza, A. H., Carvalho, V. N., Manreza, M. L. G., Mendes, M. F., Frota, E. C., Vasconcelos, C., Tumas, V., Ferraz, H. B., Barbosa, E., \& Jurno, M. E. 2015. Cannabinoids in neurology - Brazilian Academy of Neurology. Arquivos de Neuro-Psiquiatria, 73(4), 371-374.

Campos, A. C., Moreira, F. A., Gomes, F. V., Del Bel, E. A., \& Guimarães, F. S. 2012. Multiple mechanisms involved in the large-spectrum therapeutic potential of cannabidiol in psychiatric disorders. Philosophical Transactions of the Royal Society B, 367, 3364-3378.

Carlini, E. A. 2006. A história da maconha no Brasil. Jornal Brasileiro de Psiquiatria, 55(4), 314-317.

Carlini, E. A., Rodrigues, E., \& Galduróz, J. C. E. 2005. Cannabis sativa L. e substâncias canabinoides em medicina. São Paulo: CEBRID - Centro Brasileiro de Informações Sobre Drogas Psicotrópicas.

Conrad, C. 2001. Hemp: O uso medicinal e nutricional da maconha. Record: Rio de Janeiro.

Cunha, J., Carlini, E. A., Pereira, A. E., Ramos, O. L., Pimentel, C., Gagliardi, R., Sanvito, W. L, Lander, N., \& Mechoulam, R. 1980. Chronic administration of cannabidiol to healthy volunteers and epileptic patients. Pharmacology, 21, 175-185.

Crippa, J. A., Lacerda, A. L. T., Amaro, E., Busatto-Filho, G., Zuardi, A. W., \& Bressan, R. A. 2005. Efeitos cerebrais da maconha - resultados dos estudos de neuroimagem. Revista Brasileira de Psiquiatria, 27(1), 70-78.

Gonçalves, G. A. M., \& Schlichting, C. L. R. 2014. Efeitos benéficos e maléficos da Cannabis sativa. Revista Uningá Review, 20(2), 92-97.

Gontiès, B., \& Araújo, L. F. 2003. Maconha: uma perspectiva histórica, farmacológica e antropológica. Mneme-Revista de Humanidades, 4(7), 01-20.

Bergeret, J., \& Leblanc, J. 1991. Toxicomanias: uma visão multidisciplinar. Artes Médicas: Porto Alegre.

Matos, R. L. A., Spinola, L. A., Barboza, L. L., Garcia, D. R., França, T. C. C., \& Affonso, R. S. 2017. O uso do canabidiol no tratamento da Epilepsia. Revista Virtual de Química, 9(2), 786-814.

Mechoulam, R. 1973. Marijuana: chemistry, pharmacology, metabolism and clinical effects. $1^{\text {a }}$ Ed., Academic Press: New York.

Miranda R. C. 2016. O canabidiol: seu uso no Brasil. Monografia do Curso de Graduação em Farmácia Faculdade de Educação e Meio Ambiente (FAEMA), Ariquemes, 28.

Monteiro, M. 2014. Uso medicinal da maconha no Brasil fica mais próximo. Disponível em: http://m.zerohora.com.br/288/vida-e-estilo/4503232/uso-medicinal-damaconha- ${ }^{-}$no-brasil-fica-maisproximo. Acesso em: 20 mar. 2020.

Pamplona, F. A. 2014.Quais são e para que servem os medicamentos à base de cannabis? Revista da Biologia, 13(1), 28-35.

\section{Minicurrículo}

Carlos Eduardo de Morais. Graduando do Curso de Farmácia da Faculdade FIBRA.

Everson Pereira da Silva. Graduando do Curso de Gestão Pública da Unip-EAD. 
Rosa Silva Lima. Possui graduação em Licenciatura em Química pela Universidade Estadual de Goiás (2004). Especialista em Capacitação para Professora do Ensino Médio em Ciências da Natureza-Química pela Universidade de Brasília (2006). Mestre em Ciências Moleculares pela Universidade Estadual de Goiás (2010). Doutora em Química pela Universidade Federal de Goiás (2017). Professora efetiva da educação básica da Secretaria de Educação do Estado de Goiás desde 2005. Professora das disciplinas: Química Geral, Química Orgânica, Química Medicinal, Química Analítica Qualitativa e Quantitativa da Faculdade do Instituto Brasil (FIBRA) desde 2011.

Como citar: Morais, C.E., Silva, E. P., \& Lima, R.S. 2021. O uso do canabidiol como medicamento no Brasil para tratar doenças crônicas. Pubsaúde, 5, a087. DOI: https://dx.doi.org/10.31533/pubsaude5.a087

Recebido: 14 out. 2020.

Revisado e aceito: 28 out. 2020.

Conflito de interesse: os autores declaram, em relação aos produtos e companhias descritos nesse artigo, não ter interesses associativos, comerciais, de propriedade ou financeiros que representem conflito de interesse.

Licenciamento: Este artigo é publicado na modalidade Acesso Aberto sob a licença Creative Commons Atribuição 4.0 (CC-BY 4.0). 